\title{
Pharmacological inhibition of myostatin/TGF- $\beta$ receptor/pSmad3 signaling rescues muscle regenerative responses in mouse model of type 1 diabetes
}

\author{
Jaemin JEONG ${ }^{1,2, *}$, Michael J CONBOY ${ }^{1}$, Irina M CONBOY C * $^{1,}$ \\ ${ }^{1}$ Department of Bioengineering and QB3 Institute, University of California, Berkeley, Berkeley, CA 94720, USA; ${ }^{2}$ Division of Radiation \\ Oncology, Korea institute of Radiological and Medical Sciences, Seoul, Korea
}

\begin{abstract}
Aim: To study the influence of acute experimental diabetes on the regenerative potential of muscle stem (satellite) cells in mice. Methods: Male C57BL/6 young mice were injected with a single dose of streptozotocin (STZ, $180 \mathrm{mg} / \mathrm{kg}$, ip) to induce diabetes. The diabetic mice were treated with insulin $(0.75 \mathrm{U} / \mathrm{kg}$, ip), follistatin $(12 \mu \mathrm{g} / \mathrm{kg}$, im) or Alk5 inhibitor (5 $\mu \mathrm{mol} / \mathrm{L} \mathrm{per} \mathrm{kg,} \mathrm{sc)} \mathrm{once} \mathrm{a} \mathrm{day.} \mathrm{On}$ the first day when high glucose levels were found, cardiotoxin (CTX) was focally injected into tibialis anterior and gastronemius muscles of the mice. The muscles were harvested $3 \mathrm{~d}$ and $5 \mathrm{~d}$ after CTX injection, and myofibers and satellite cells were isolated. Quantitative ex-vivo and in-vivo assays of myogenic potential were used to evaluate the muscle regenerative responses.

Results: The satellite cells from the diabetic mice $3 \mathrm{~d}$ after CTX injection fail to activate, and the repair of muscle deteriorates, resembling that observed in old control mice. Furthermore, the satellite cells have excessive levels of myostatin, TGF- $\beta$ receptor 1 , pSmad3 and the cell cycle inhibitor p15, while the level of TGF- $\beta 1$ remain unchanged. Treatment of the diabetic mice with insulin rescued muscle regenerative responses, and restored the expression levels of myostatin, TGF- $\beta$ receptor 1 , pSmad3, and p15 to those similar of healthy controls. Treatment of the diabetic mice with the myostatin antagonist follistatin, or with the Alk5 inhibitor of TGF- $\beta$ receptor 1 (which did not diminish the blood glucose levels) rescued muscle regenerative responses and attenuated the myostatin/ TGF $\beta$ receptor/pSmad3 signaling.
\end{abstract}

Conclusion: The muscle regenerative responses are incapacitated and repair of the tissue fails within hours after the initiation of hyperglycemia in a mouse model of type 1 diabetes, but stem cell function is rescued by insulin, as well as follistatin or an Alk5 inhibitor that blocks TGF- $\beta$ receptor signaling.

Keywords: diabetes; muscle regeneration; stem cell; satellite cell; cardiotoxin I; insulin; follistatin; Alk5 inhibitor; myostatin; TGF- $\beta$ receptor; pSmad signaling

Acta Pharmacologica Sinica (2013) 34: 1052-1060; doi: 10.1038/aps.2013.67; published online 17 Jun 2013

\section{Introduction}

The function of pancreatic $\beta$-cells is indispensable for the production of insulin and thus for the regulation of glucose homeostasis. Autoimmune, type 1 diabetes mellitus (diabetes) depletes pancreatic $\beta$-cells. The non-autoimmune dysfunction of these cells or peripheral insulin resistance is manifested as type 2 diabetes; both types result in a number of mild and severe metabolic disorders along with of course increased blood glucose levels ${ }^{[1,2]}$. Diabetic patients also may suffer

\footnotetext{
${ }^{*}$ To whom correspondence should be addressed.

E-mail iconboy@berkeley.edu (Irina M CONBOY); jmj1103@kirams.re.kr (Jaemin JEONG)

Received 2012-12-13 Accepted 2013-04-25
}

a lack of cell and organ function, poor wound healing and reduced longevity due to dysfunctions in many organ systems $^{[1,3]}$. The deregulation of glucose metabolism in diabetes causes a plethora of negative changes in the endocrine system, cardio-vascular system, adipose tissue, liver, etc, which may indirectly affect the general success of organ repair, both individually and cumulatively. As tissue maintenance, repair and healing are mediated by adult stem and progenitor cells, we asked what effects diabetes might have on the function of these crucial cells and how long would it take for the regenerative responses to decline following the onset of hyperglycemia.

The specific focus of this work was on muscle stem cells (satellite cells), which reside beneath the basal lamina of muscle 
fibers and are responsible for the maintenance and repair of skeletal muscle throughout adult life ${ }^{[4-6]}$. Our results demonstrate that when insulin deficiency is induced through the depletion of pancreatic $\beta$-cells by streptozotocin $(S T Z)^{[7]}$, the regenerative responses of muscle stem cells (as measured as the ability to give rise to proliferating fusion-competent myoblasts), quickly deteriorate in vivo, and muscle fails to repair after injury. While STZ has been previously described to have an inhibitory effect on the fusion of cultured myoblasts, which was not rescued by exogenous insulin ${ }^{[8]}$, in our hands, the myogenicity of satellite cells and tissue regeneration was restored to diabetic animals by systemic administration of insulin, demonstrating that in vivo the effects of one STZ administration is transient to myoblasts and specific to the ablation of insulin-producing $\beta$-islet cells. Notably, we found that STZ inhibits satellite cell responses by inducing Myostatin/pSmad3 signaling, and that follistatin or an Alk5 inhibitor (a small molecule inhibitor of TGF- $\beta$ receptor 1 ), rescues satellite cell responses and improves muscle repair while in the diabetic condition, suggesting promising avenues for improving tissue regeneration in diabetic patients. Myostatin mRNA was previously found to increase in mice treated with STZ ${ }^{[9]}$ and myostatin is a known inhibitor of satellite cell proliferation ${ }^{[10]}$, however, the in vivo effects of experimental diabetes on muscle stem cells by increased TGF- $\beta$ receptor signaling (including elevated pSmad3 and p15), and the rescue of muscle repair in vivo by pharmacological inhibitors of this pathway are, to our knowledge, entirely novel findings of this work.

While STZ-induced diabetes was previously demonstrated to result in smaller and weaker skeletal muscle ${ }^{[11,12]}$, the muscle cell biology, especially the effects of this molecule on satellite cells in vivo, was not described. When primary muscle cell lines are established from mice that model diabetes or from humans with type 2 diabetes, their ability to fuse into myotubes in culture does not decline as compared to the control cell lines ${ }^{[8]}$. However, these primary cells are certainly different from the stem cells in vivo, not only because the expression of numerous genes rapidly changes in culture, but also because culture conditions (media and adhesion substrates), do not faithfully mimic the local and systemic in vivo environment, and particularly that present in cases of diabetes. Hence, in this work we addressed the effects of diabetes on muscle regenerative potential in pharmacological and genetic mouse models, and uncovered that acute experimental diabetes induces myostatin, which in turn inhibits the activation of muscle stem cells, causing poor muscle repair.

\section{Materials and methods}

Mice

Young (2-4 month) and C57BL/6J-Ins2Akita male mice were obtained from Jackson Laboratories (Bar Harbor, ME, USA), and old (20-24 month) C57BL/6 male mice were obtained from the NIA (Bethesda, MD, USA). Animals were housed and cared for under the UC Berkeley Office of Laboratory Animal Care, with protocols approved by the UC Berkeley Animal Care and Use Committee.
STZ, insulin, follistatin (FSTN) and TGF $\beta$ receptor 1 (Alk5) inhibitor administration

Young mice were given a single intraperitoneal injection (ip) of STZ (Sigma-Aldrich, MO, USA) at a dose of $180 \mathrm{mg} / \mathrm{kg}$ body weight, or control buffer injection. After one week of STZ treatment, blood glucose levels were measured using OneTouch UltraMini (LifeScan, CA, USA). Animals showing blood glucose level $>300 \mathrm{mg} / \mathrm{dL}$ were considered hyperglycemic. Hyperglycemic experimental mice were given intraperitoneal injections of insulin $(0.75 \mathrm{U} / \mathrm{kg}$, Sigma-Aldrich, $\mathrm{MO}$, USA), and/or intramuscular injection of FSTN $(12 \mu \mathrm{g} / \mathrm{kg}$, R\&D systems, MN, USA), and/or subcutaneous injection of Alk5 inhibitor ( $5 \mu \mathrm{mol} / \mathrm{L}$ per $\mathrm{kg}$, Calbiochem, NJ, USA) once a day after the induction of hyperglycemia.

\section{C57BL/6J-Ins2Akita mice}

This strain of mice (Jackson Laboratories) was used for the studies of myogenic potential in the same assays as the wild type young mice. Blood glucose levels were measured using OneTouch UltraMini (LifeScan, CA, USA). These animals showed high blood glucose levels (520-583 mg/dL), indicating they were hyperglycemic. Some of these animals received intraperitoneal injections of insulin $(0.75 \mathrm{U} / \mathrm{kg}$, Sigma-Aldrich, MO, USA), resulting in normal blood glucose levels (184-193 $\mathrm{mg} / \mathrm{dL}$ ) by 1-h post injection.

\section{Muscle injury}

Isoflurane was used to anesthetize the animals during the muscle injury procedure. For bulk myofiber and satellite cell activation, tibialis anterior and gastrocnemius muscles were injected with cardiotoxin I (CTX, Sigma-Aldrich, MO, USA) dissolved at $100 \mu \mathrm{g} / \mathrm{mL}$ in PBS, at 2-5 sites of $10 \mu \mathrm{L}$ for each muscle. Muscles were then harvested $3 \mathrm{~d}$ later. For focal injury, to assay regeneration in vivo, 2-3 $\mu \mathrm{L}$ of $1 \mu \mathrm{g} / \mathrm{mL}$ CTX was injected at one site to the middle of the tibialis anterior or two sites to the gastrocnemius, and muscle harvested 5 days post injury (DPI).

\section{Isolation of myofibers and satellite cells}

Myofibers and satellite cells were purified as described ${ }^{[13]}$. Briefly, dissected muscle was digested for $1 \mathrm{~h}$ and $30 \mathrm{~min}$ at $37^{\circ} \mathrm{C}$ in DMEM with $1 \%$ penicillin-streptomycin, 250 units $/ \mathrm{mL}$ Collagenase Type IIA (Sigma-Aldrich, MO, USA) and dissociated into myofibers with attached satellite cells by trituration, sedimentation and washing. Satellite cells were liberated from the fibers by digestion for $1 \mathrm{~h}$ in DMEM with $40 \mathrm{U} / \mathrm{mL}$ Collagenase type II and $2 \mathrm{U} / \mathrm{mL}$ Dispase, followed by sedimentation, filtration and washing. Leukocytes were depleted using anti-CD45-coated magnetic beads (Miltenyi Biotech). This procedure yields $\sim 95 \%$ pure satellite cells, based on their functional activity ${ }^{[13,14]}$.

\section{Satellite cell myogenic potential}

Assays of myogenic potential were performed, as previously described $^{[13]}$. Briefly, activated in vivo myofiber-associated satellite cells were cultured in growth medium on ECM:DMEM- 
coated plates (1:500; BD Biosciences, CA, USA) for $48 \mathrm{~h}$. Growth medium (GM) was composed of Ham's F10 (Mediatech, VA, USA), 20\% bovine growth serum (HyClone, IL, USA), $5 \mathrm{ng} / \mathrm{mL}$ bFGF (Invitrogen, CA, USA), and 1\% penicillin/ streptomycin (Invitrogen, CA, USA). GM was replaced daily. After addition of BrdU $(10 \mu \mathrm{mol} / \mathrm{L}$, Sigma-Aldrich, MO, USA $)$ to the GM for $2 \mathrm{~h}$ to label proliferating cells, the cultures were switched into differentiation medium (DM, DMEM with 2\% horse serum) for $48 \mathrm{~h}$, in order to assay generation of de-novo myotubes, which are typically produced by fusion-competent myoblasts in DM.

\section{Muscle repair assays by immunofluorescence and histological analysis}

All muscle tissue was dissected, flash frozen in OCT compound (Tissue Tek, Japan), cryo-sectioned at $10 \mu \mathrm{m}$, as previously described ${ }^{[13]}$. Muscle sections were stained with aqueous hematoxylin and eosin (H\&E; Sigma-Aldrich, MO, USA) and immunostaining was performed as described ${ }^{[13]}$. Briefly, after permeabilization in PBS $+1 \%$ FBS $+0.25 \%$ Triton-X-100, tissues and cells were incubated with $1 \mu \mathrm{g}$ of primary antibodies per milliliter of staining buffer (PBS+1\% FBS) for $1 \mathrm{~h}$ at room temperature, followed by washes with staining buffer and then by $1 \mathrm{~h}$ incubation with a $1 \mathrm{\mu g} / \mathrm{mL}$ dilution of secondary, fluorochrome labeled antibodies (ALEXA, Invitrogen, CA, USA) in staining buffer. BrdU-specific immunostaining required an extra step of $2 \mathrm{~mol} / \mathrm{L} \mathrm{HCl}$ treatment (for denaturation/nicking of DNA) before the permeabilization step.

\section{Western blot analysis}

Western blot analysis was conducted as described ${ }^{[15]}$. Briefly, myofiber and satellite cell lysate were prepared in RIPA lysis buffer $(50 \mathrm{mmol} / \mathrm{L}$ Tris- $\mathrm{HCl}, \mathrm{pH} 7.5,150 \mathrm{mmol} / \mathrm{L} \mathrm{NaCl}, 1 \%$ Triton X-100, 1\% sodium-deoxycolate, $1 \mathrm{mmol} / \mathrm{L}$ EDTA and $0.1 \%$ SDS) as described. Fifty micrograms proteins were run on SDS PAGE gels (Bio-Rad, CA, USA). Anti-TGFß1 (1:100, Santa Cruz Biotech, CA, USA), myostatin (1:500, Santa Cruz Biotech, CA, USA), p15 (1:100, Santa Cruz Biotech, CA, USA), pSMAD 2, 3 (1:100, Santa Cruz Biotech, CA, USA), SMAD 3 (1:100, Abcam, MA, USA), total actin (A5060, Sigma, MO, USA) antibodies were diluted in 5\% skim milk in $1 \times$ PBST and incubated for overnight at $4{ }^{\circ} \mathrm{C}$. HRP conjugated secondary antibodies (1:5000, Santa Cruz Biotech, CA, USA) were incubated for $1 \mathrm{~h}$ at room temperature and developed using ECL plus Western blotting detection system (GE Healthcare), and imaged on a Bio-Rad Chemidoc XRS.

\section{Statistical analysis}

Quantified data are expressed as mean \pm standard deviation. Statistical significance was determined, using one-way ANOVA of variance to compare data from different experimental groups. A minimum of three independent experiments (eg, 3 control vs $3 \mathrm{STZ}$ administered animals) were performed for each described condition. ${ }^{\mathrm{b}} P<0.05$ and ${ }^{\mathrm{c}} P<0.01$ were considered statistically significant.

\section{Results}

Altered glucose metabolism negatively influences muscle stem cell performance

Experimental diabetes was induced through depletion of pancreatic $\beta$-cells by STZ in young C57BL/ 6 male mice. Blood glucose levels were measured daily and on the first day of high glucose levels $(375-497 \mathrm{mg} / \mathrm{dL})^{[16]}$ hind leg muscle (Tibialis Anterior, TA and Gastronemius, Gastroc) of the mice was injured by injection of cardiotoxin (CTX). Seventy-two hours after injury, the activated satellite cells were assessed for their regenerative capacity by their ability to form myogenic cultures ex-vivo overnight, and at 5 days post injury (DPI) muscle was assayed for the success in early regeneration by the regeneration of injured tissue with newly formed myofibers, using our conventional and previously published methods ${ }^{[4,13,14,17,18]}$. As shown in Figure 1A, satellite cells from muscle of untreated control mice (with blood glucose levels of $175-190 \mathrm{mg} / \mathrm{dL})^{[16]}$ rapidly and robustly produced $\mathrm{eMyHC}^{+}$de-novo myofibers. In dramatic contrast, just $72 \mathrm{~h}$ after the loss of insulin and elevation of blood glucose levels, the regenerative responses of young satellite cells deteriorated and became similar to those derived from old mice (Figure 1A, quantified in 1B).

These results demonstrate that the regenerative responses of muscle stem cells rapidly decline in vivo upon insulin loss and induced hyperglycemia, such that these cells fail to generate proliferating fusion-competent myoblasts ex-vivo, in tissue explants (myofibers with their associated satellite cells). While normal insulin and glucose levels are present in vitro, it is the in vivo myogenic activation of satellite cells that is measured by this assay, eg the overnight formation of myogenic colonies by the satellite cells, which were activated by muscle injury (or not), in vivo for $3 \mathrm{~d}^{[19]}$.

In agreement with these data, a profound decline in muscle repair was manifested in STZ-treated young mice, as compared with control animals. eMyHC ${ }^{+}$myofibers with centrally-located nuclei did not efficiently form in STZ-treated animals, and instead areas of scarring and inflammation replaced the damaged tissue, which are also phenotypes that are typically observed during the poor repair seen in old mice $\mathrm{e}^{[4,13,17]}$ (Figure 1C, quantified in 1D).

\section{Exogenous insulin restores the satellite cell myogenicity and muscle regeneration in hyperglycemic mice}

To rule out non-specific effects of STZ and to confirm experimental diabetes as the cause of decline in myogenic responses, we rescued both the in vitro and in vivo myogenicity by the administration of insulin to STZ-treated mice (Figure 2A-2D). The blood glucose levels were normalized from 375-497 $\mathrm{mg} / \mathrm{dL}$ to $173-190 \mathrm{mg} / \mathrm{dL}$ by daily single injections of insulin that continued during the entire period of muscle regeneration. However, by $24 \mathrm{~h}$ after each injection hyperglycemia returned, which explains the robust yet incomplete $(\sim 80 \%)$ rescue of myogenicity by insulin, and emphasizes that even transient experimental diabetes significantly inhibits muscle regeneration. 
A

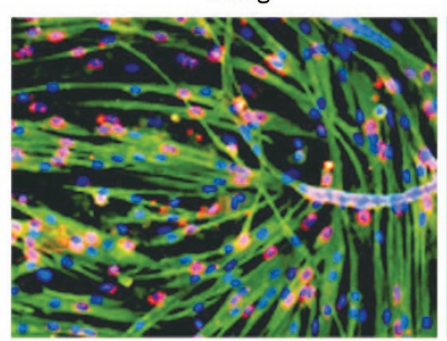

Young/STZ

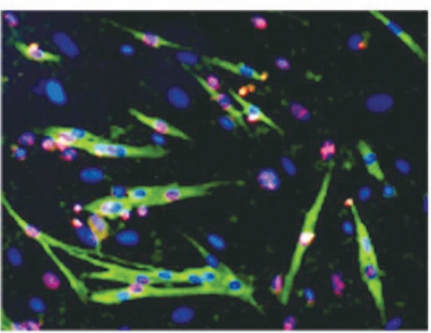

eMyHC/BrdU/Hoechst
Old

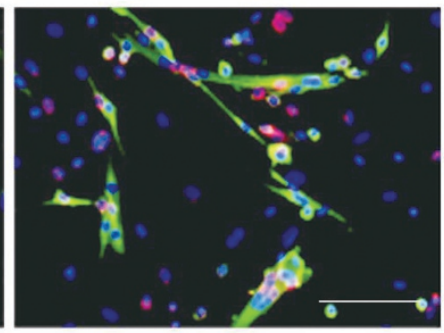

B

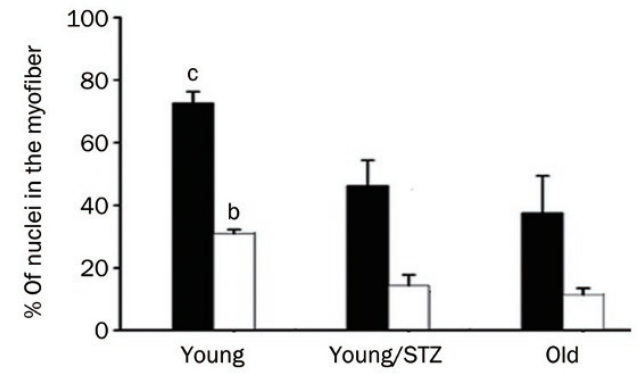

D

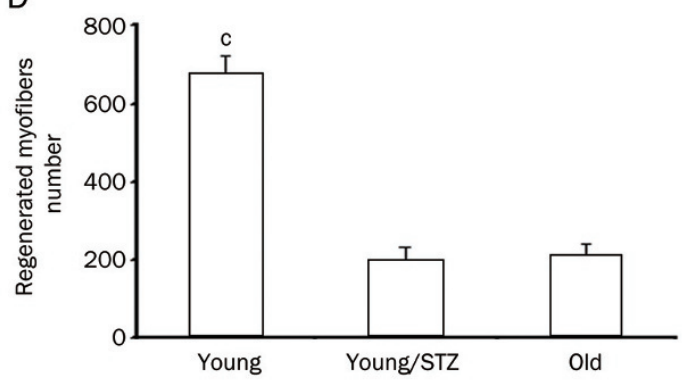

C

H\&E
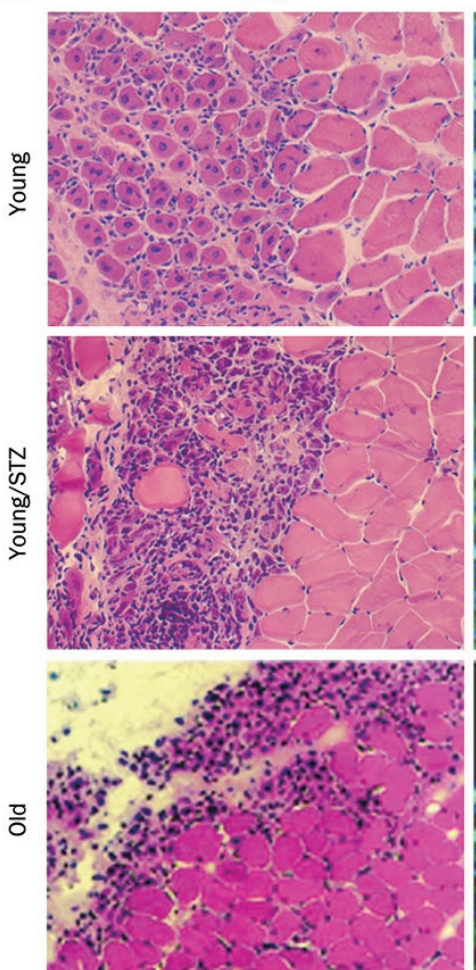
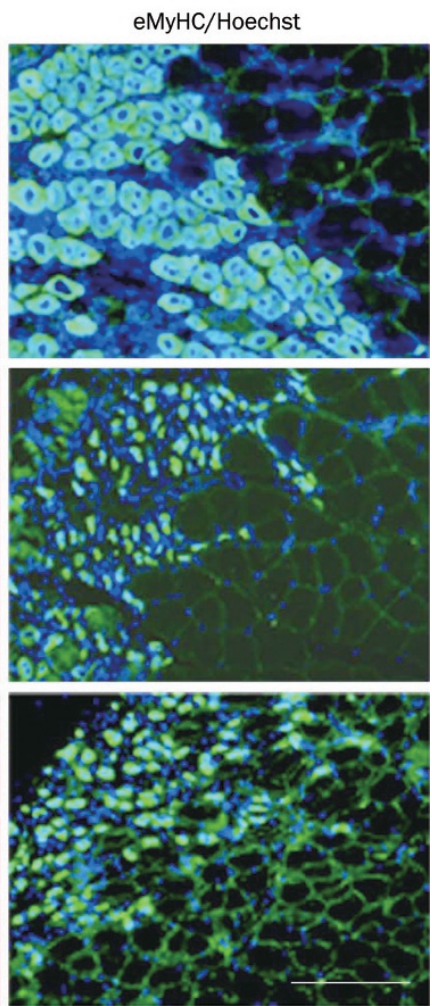

Figure 1. Satellite cell myogenic potential and muscle regeneration in vivo declines in STZ treated young mice due to hyperglycemia. (A) Satellite cells were prepared from tibialis anterior (TA) and gastrocnemius (Gastroc) muscle of young, STZ treated young, and old control mice at 3 days post-injury (DPI), cultured in growth medium for $48 \mathrm{~h}$ and then switched to differentiation medium for $48 \mathrm{~h}$. Formation of de-novo eMyHC ${ }^{+} \mathrm{myotubes}(\mathrm{green})$ and $\mathrm{BrdU}^{+}$ nuclei (red) was assayed by immunofluorescence. Hoechst (blue) labels all nuclei. Scale bar, $100 \mu \mathrm{m}$. (B) The percent of nuclei in eMyHC ${ }^{+}$myotubes to total nuclei (black bars), and the percent of $\mathrm{eMyHC}^{+}$myotubes with more than two nuclei to total nuclei (white bars) were quantified. $n=3$ with $>3$ independent experiments; ${ }^{b} P<0.05$, and ${ }^{c} P<0.01$ Young as compared to STZ treated young. Data are mean \pm SD. (C) Assessment of success in muscle regeneration in vivo was performed by hematoxylin and eosin (H\&E) histological analysis and by immunodetection of de-novo eMyHC ${ }^{+}$myofibers (green) in $10 \mu \mathrm{m}$ cryosections of TA and Gastroc muscle that were isolated at 5 DPI. Scale bar, $100 \mu \mathrm{m}$. (D) Numbers of de-novo myofibers per mm ${ }^{2}$ of injured muscle (covering the entire injury site) were quantified. $n=3$ with $>3$ independent experiments; ${ }^{c} P<0.01$ Young as compared to STZ-treated young muscle. Data are mean \pm SD.

To analyze in more detail the inflammatory response in the injured muscle, we immunostained muscle sections derived from control and STZ treated mice with CD11b (a macrophage marker) and eMyHC (Supplementary Figure 1). These results confirmed that the presence of macrophages in the injured muscle of STZ mice was much more pronounced as compared to the untreated control mice and that insulin rescue treatment reduced the tissue inflammation (Supplementary Figure 1).
Experimental diabetes causes an up-regulation of myostatin and of TGF $\beta$ receptor 1 in satellite cells

To address the molecular mechanism by which experimental diabetes causes the decline in muscle regeneration, we focused on TGF- $\beta$ / pSmad signaling, since it has been previously found that excessive signaling through this pathway is capable of preventing the activation and proliferation of satellite cells ${ }^{[10,15,20]}$. To investigate if acute experimental dia- 
A

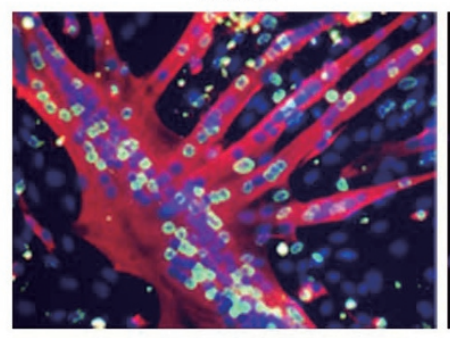

STZ

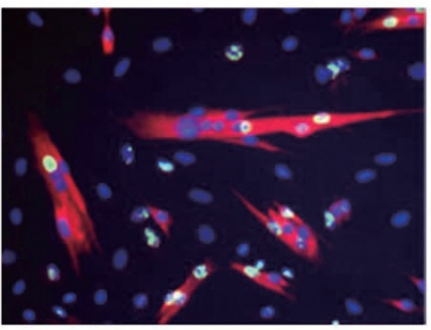

eMyHC/BrdU/Hoechst

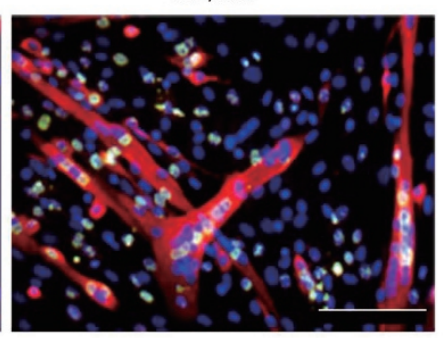

STZ/INS

C
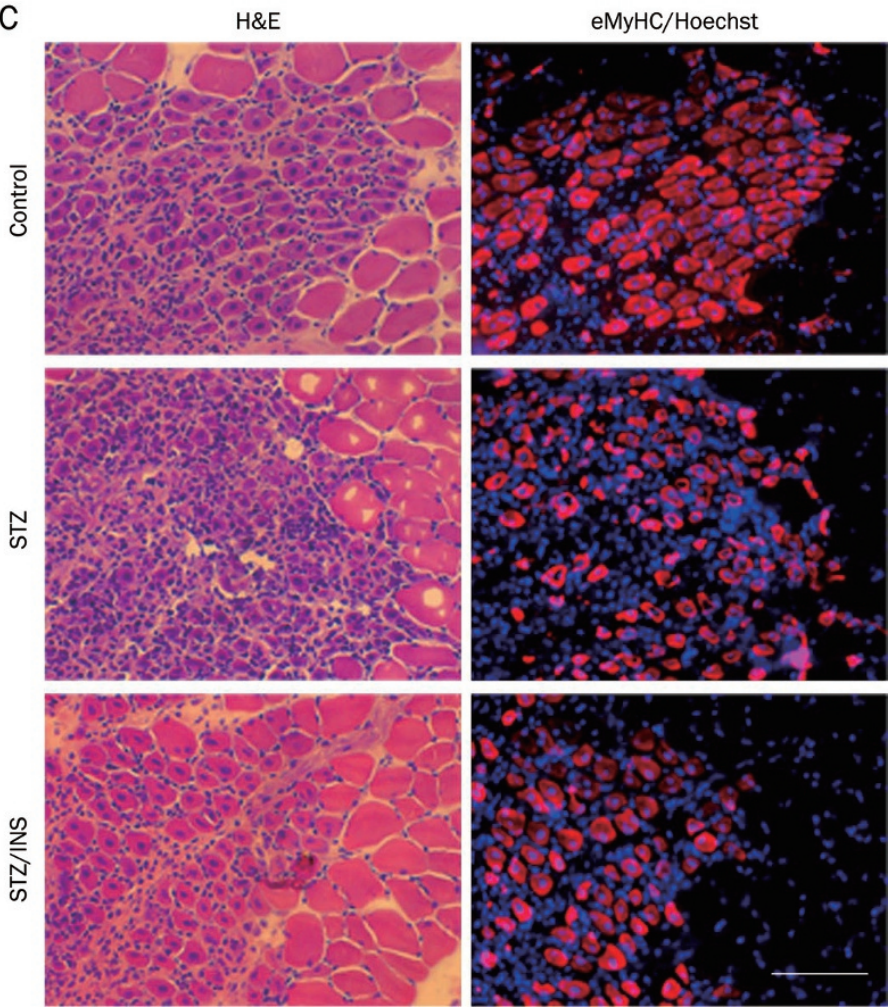

Figure 2. Insulin rescues myogenic responses in hyperglycemic by restoring glucose metabolism. (A) Satellite were prepared from control young mice that were treated with STZ or STZ and rescued by insulin (STZ/INS), and their myogenic potential was determined ex-vivo, as in A, quantified in B. $n=3$ with $>3$ independent experiments. ${ }^{c} P<0.01$ Control as compared to STZ, and STZ as compared to STZ/INS. Data are mean \pm SD. (B) The percent of nuclei in $\mathrm{eMyHC}^{+}$myotubes relative to total nuclei (black bars), and the percent of eMyHC myotubes with more than two nuclei relative to tatal cel number (white bars) were quantified. $n=3$ with $>3$ independent experiments. (C) A comparison of success in muscle regeneration in vivo was performed between the Control, STZ, and STZ/INS mice, as in C, quantified in D. $n=3$ with $>3$ independent experiments. ${ }^{\circ} P<0.01$ Control as compared to STZ and ${ }^{\mathrm{b}} \mathrm{P}<0.05 \mathrm{STZ}$ as compared to STZ/INS. Satellite cells from STZ-treated mice displayed diminished myogenic capacity in vitro and in vivo, and insulin administration rescued the regenerative responses of these muscle stem cells, demonstrating that the decline in myogenicity was caused by hyperglycemia.

betes causes the rapid deterioration of satellite cell responses through an increase in TGF $\beta$ signaling, we analyzed the expression of the ligands myostatin and TGF $\beta 1$, and of the TGF $\beta$ receptor 1 in satellite cells that were lysed for Western blotting immediately after isolation from regenerating hind leg muscle. As shown in Figure 3A (quantified in 3B) and Figure $4 \mathrm{E}$ (quantified in $4 \mathrm{~F}$ ), the protein levels of TGF $\beta$ receptor 1 and the ligand myostatin, phosphorylated Smad3 (pSmad3, a downstream effector of TGF $\beta$ receptor signaling ${ }^{[21]}$, and the cell cycle inhibitor p15 (a known transcriptional target of $\mathrm{TGF} \beta / \mathrm{pSmad}$ signaling ${ }^{[22]}$, are all increased in STZ treated, as compared to control mice, while the levels of the TGF- $\beta 1$ remain unchanged. In agreement with the enhancement of myogenesis, the expression of TGF $\beta$ receptor 1 , myostatin, pSmad 3 and p15 were down-modulated back to normal in satellite cells derived from STZ treated mice rescued with insulin (Figure $3 \mathrm{~A}$ and $4 \mathrm{E}$ ). These data demonstrate that insulin reversed the molecular effects of STZ and normalized TGF- $\beta$ 
A

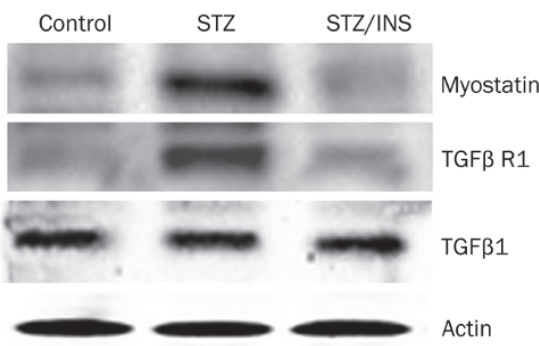

B

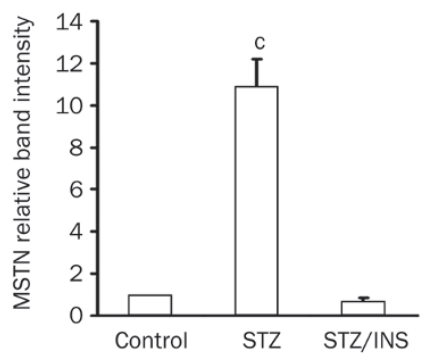

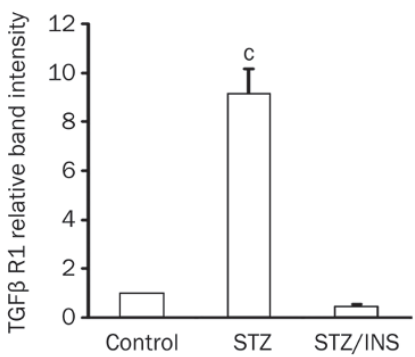
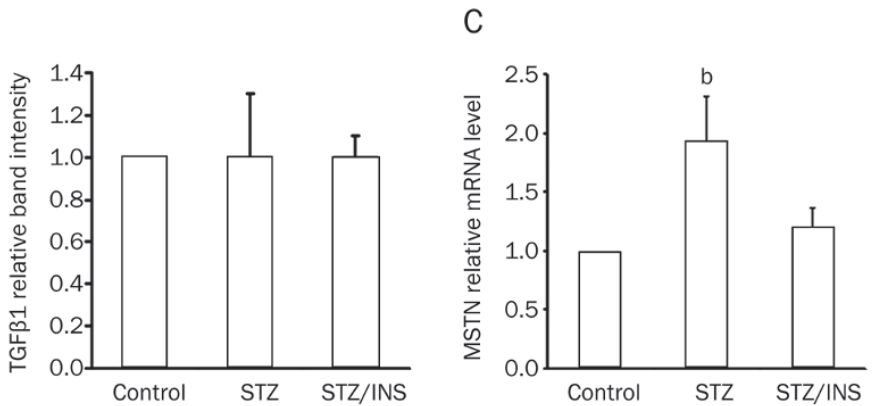

Figure 3. Myostatin and TGF $\beta$ receptor 1, but not TGF 1 ligand, levels are increased in STZ-treated mice due to hyperglycemia. (A) Satellite cells were prepared from TA and Gastroc muscle of Control, STZ and STZ/INS treated mice at 3 DPI, lysed right after their isolation from hind leg muscle and immunoblotted for myostatin, TGF $\beta$ and TGF $\beta$ receptor 1. Actin was used as a loading control. (B) The quantification of protein band intensity normalized by actin was performed. $n=3$ with $>3$ independent experiments; ${ }^{\circ} P<0.01$ Control and STZ/INS as compare to STZ. Data are mean $\pm S D$. (C) qRT PCR was performed on these satellite cells, using primers that are specific for myostatin. $n=3$ with $>3$ independent experiments; ${ }^{b} P<0.05$ Control as compare to STZ. STZ treatment up-regulated the expression of myostatin and TGF $\beta$ receptor 1, but not TGF $\beta$, while administration of insulin reduced the expression levels of these genes back to those typical of young control mice, demonstrating that excessive gene expression was caused by hyperglycemia.

receptor/myostatin signaling and the levels of p15 in satellite cells of STZ-treated animals, which confirms that the deregulation of TGF- $\beta$ signaling was indeed caused by experimental diabetes and not by any other effect of STZ administration.

To look more closely at the mechanism by which experimental diabetes induces myostatin protein levels, we analyzed the expression of myostatin mRNA in the satellite cells, using qRT-PCR (Figure 3C). These data clearly showed that mRNA levels of myostatin were significantly up-regulated in STZ treated mice and were attenuated in STZ-treated mice rescued with insulin, in strong correlation with the protein expression (Figure 3C). These results suggest that in hyperglycemic STZtreated mice, the canonical TGF $\beta$ receptor signaling pathway becomes over-activated, which causes hyper-phosphorylation of Smad3 and induction of the p15 cell cycle inhibitor in muscle stem cells.

Inhibition of TGF $\beta$ receptor signaling by follistatin rescues the regenerative responses of muscle stem cells in hyperglycemic mice

To confirm and extrapolate these data, we attempted to rescue muscle regeneration in vivo, and the muscle stem cell responses in vitro, by systemic administration of follistatin, a natural antagonist of myostatin, and by administration of the Alk5 inhibitor of TGF $\beta$ receptor 1 . As shown in Figure $4 \mathrm{~A}, 4 \mathrm{C}$ (quantified in $4 \mathrm{~B}, 4 \mathrm{D}$ ), the formation of new eMyHC $\mathrm{C}^{+}$ myofibers in vivo and of $\mathrm{eMyHC}^{+}$primary myotubes in vitro were restored in STZ-treated mice by the in vivo administra- tion of these TGF $\beta$ pathway inhibitors. Notably, the levels of blood glucose remained high in Alk5 inhibitor treated animals $(349-473 \mathrm{mg} / \mathrm{dL}$ ) and similarly, single intramuscular injection of follistatin is not expected to change blood glucose levels; however, the muscle regenerative capacity was much improved, suggesting that up-regulation of the TGF $\beta / \mathrm{pSmad}$ pathway was the main inhibitory culprit for the lack of satellite cells responses in this STZ model of type 1 diabetes.

\section{Muscle regeneration fails in hyperglycemic mice due to pSmad 3-affected up-regulation of $\mathrm{p} 15$}

To confirm that the rescue of myogenic responses in STZ-mice was indeed caused by an attenuation of TGF $\beta / p S m a d$ signaling, we performed Western blotting on satellite cells which were lysed immediately after their isolation from regenerating hind leg muscle of control, STZ-treated, STZ-treated with insulin rescue, and STZ/Alk5 inhibitor-treated mice. Immunoblotting was performed with antibodies that are specific for phosphorylated Smad3 (pSmad3), and p15. Actin was used as a loading control. As shown in Figure 4E (quantified in $4 \mathrm{~F})$, both Alk5 inhibitor and insulin significantly diminished the levels of pSmad 3 and $\mathrm{p} 15$ proteins in satellite cells in vivo, demonstrating that the canonical TGF- $\beta /$ pSmad3 pathway becomes elevated by experimental diabetes.

Up-regulation of myostatin in genetic model of type 1 diabetes, Akita mice

To confirm our conclusions using a genetic model of type 1 
A
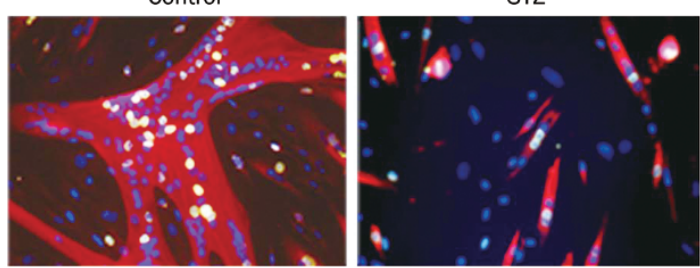

B

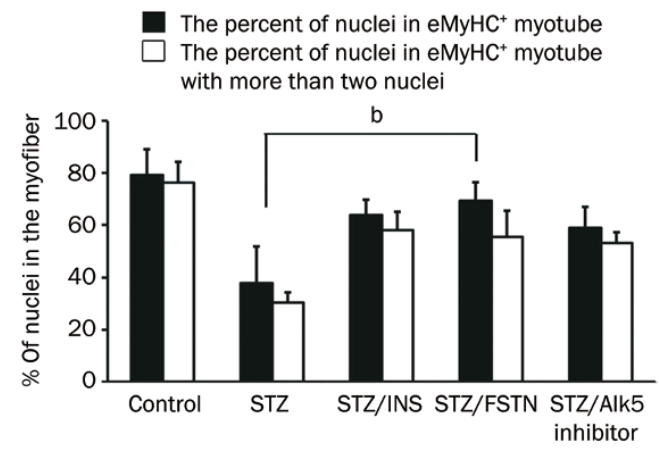

C
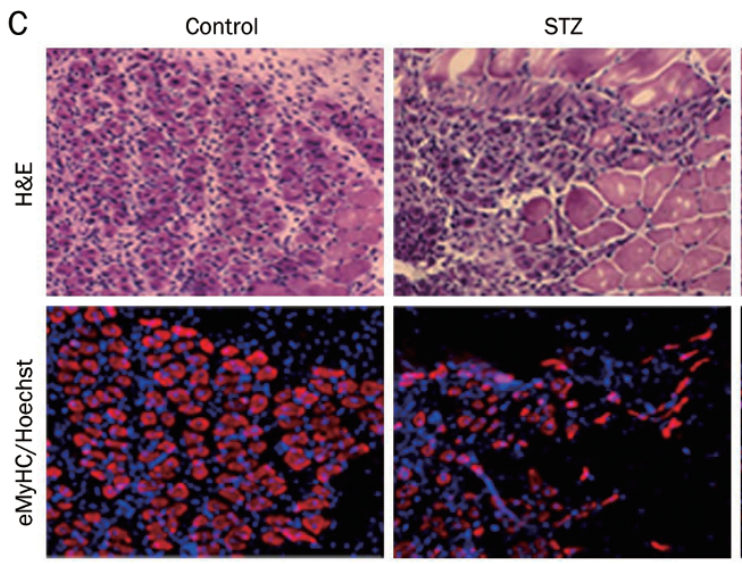

E

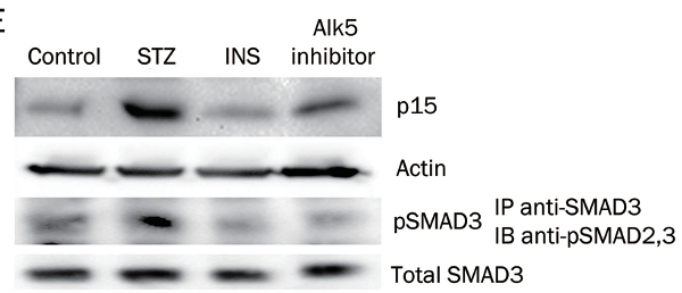

STZ/INS

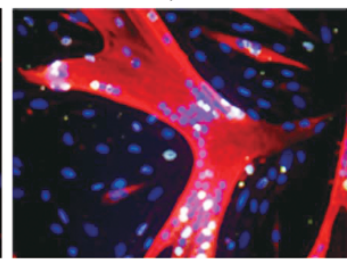

eMyHC/BrdU/Hoechst
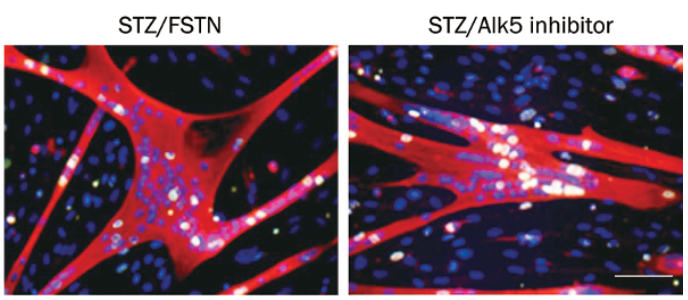

D
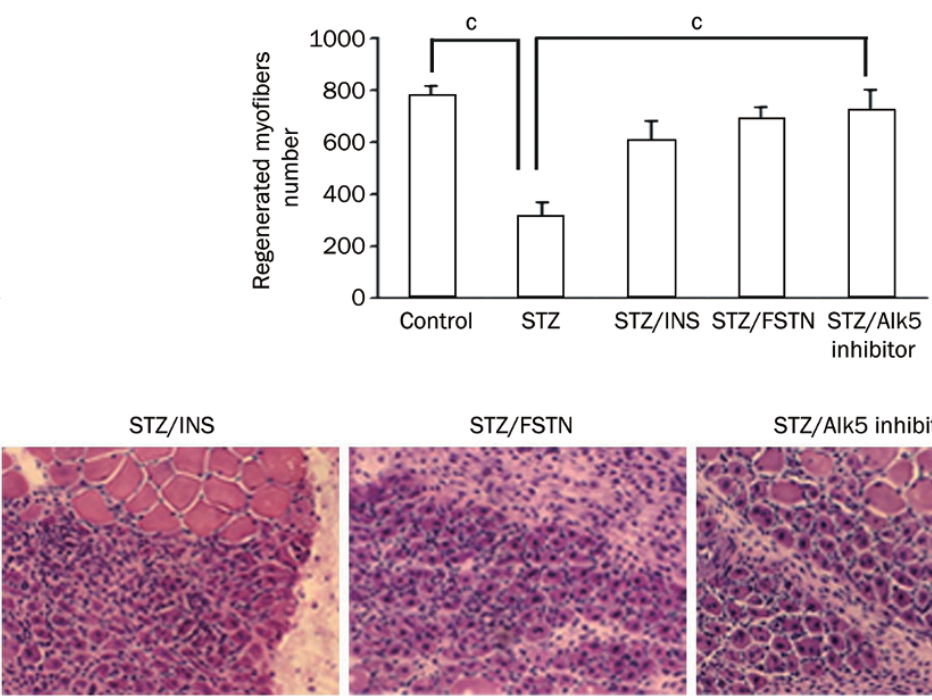

STZ/Alk5 inhibitor
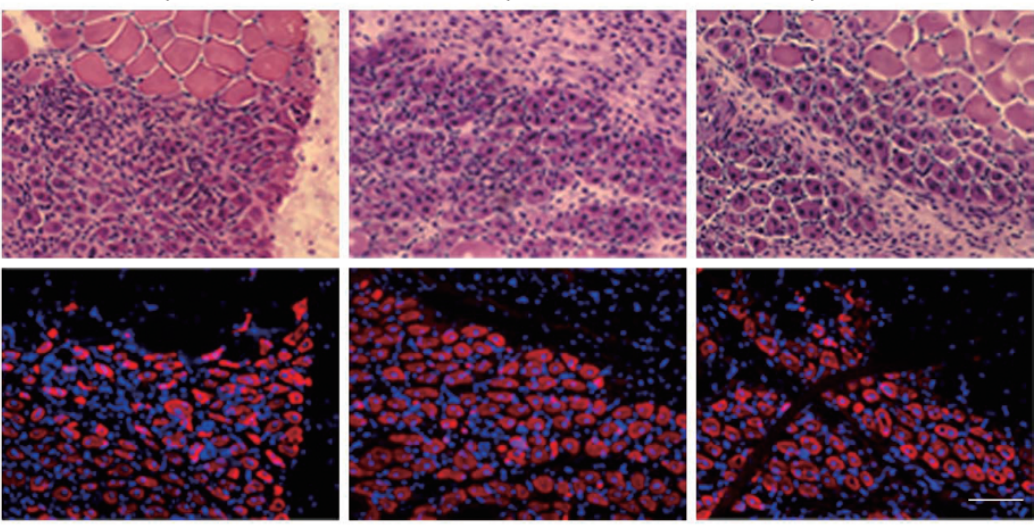

F
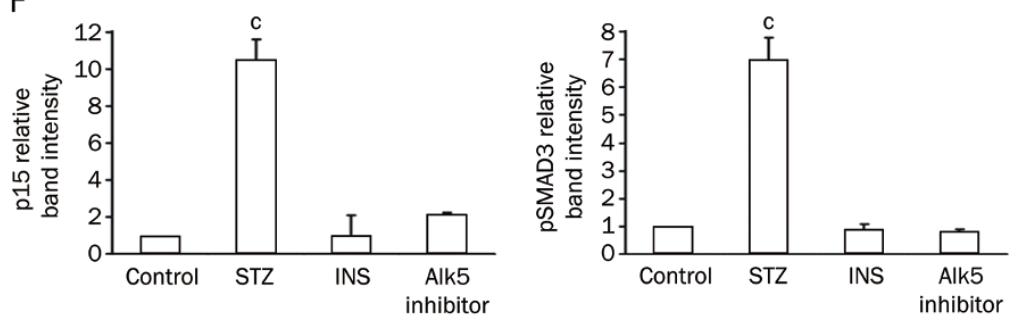

Figure 4. Regenerative capacity of muscle stem cells is rescued in STZ treated mice by the attenuation of TGFB/pSmad signaling intensity. (A) Muscle fibers were prepared from TA and Gastroc muscle of control, STZ, STZ/INS, STZ/FSTN (treated with follistatin) and STZ/Alk5 inhibitor treated mice at $3 \mathrm{DPI}$, cultured in growth medium for $48 \mathrm{~h}$ and then switched to differentiation medium for $48 \mathrm{~h}$. Formation of de-novo eMyHC ${ }^{+}$myotubes (red) was assayed by immunofluorescence. Hoechst (blue) labels all nuclei. Scale bar, $100 \mu \mathrm{m}$. (B) The ratio of the nuclei in eMyHC ${ }^{+}$myotubes to total nuclei 100 (black bars), and the ratio of the eMyHC ${ }^{+}$myotubes with than two nuclei to total nuclei $\times 100$ (white bars) were quantified. $n=3$ with $>3$ independent experiments. ${ }^{b} P<0.05 \mathrm{STZ}$ as compared to FSTN. Data are mean \pm SD. (C) An assessment of success in muscle regeneration in vivo was performed by hematoxylin and eosin (H\&E) histological analysis and by immunodetection of de-novo eMyHC ${ }^{+}$myofibers (red) in $10 \mu \mathrm{m}$ cryosections of TA and Gastroc muscle that were isolated at 5 DPI. INS, FSTN, and Alk5 inhibitor treated muscle had significantly improved regeneration. Scale bar, 100 $\mu \mathrm{m}$. (D) Numbers of de-novo myofibers per $\mathrm{mm}^{2}$ of injured muscle (covering the entire injury) were quantified. $n=3$ with $>3$ independent experiments; ${ }^{\circ} P<0.01$ Control as compare to STZ, and STZ as compared to Alk5 inhibitor. Data are mean \pm SD. (E) Satellite cells were prepared from TA and Gastroc muscle of Control, STZ, INS, FSTN, and Alk5 inhibitor treated mice at 3 DPI, lysed right after their isolation from hind leg muscle and immunoblotted for p15, phosphorylated Smad3 and total Smad3. Actin was used as a loading control. The levels of $\mathrm{p} 15$ and pSmad 3 were upregulated in cells that were derived from STZ-treated mice and were normalized by the in vivo administration of insulin or Alk5 inhibitor. (F) The quantification of protein band intensity. $n=3$ with $>3$ independent experiments. ${ }^{c} P<0.01$ Control and INS as compare to STZ. Data are mean \pm SD. 
diabetes, we also conducted studies with C57BL/6J-Ins2Akita mice that are heterozygous for the Akita spontaneous mutation $^{[23]}$. With respect to studying muscle regeneration, there is no perfect genetic model of diabetes, since inactivation of insulin 2 can be compensated for by the up-regulation of insulin 1 in Akita mice ${ }^{[24]}$ and because NOD mice become diabetic later in life when the muscle regenerative capacity of wild type mice also declines ${ }^{[25]}$. Nevertheless, as shown in Figure 5, in Akita mice as compared with wild type C57BL/6J mice, muscle regeneration becomes diminished and the expression of myostatin is elevated. These findings are consistent with our conclusion that experimental diabetes causes an increase in myostatin, which consequentially inhibits muscle regeneration. Additionally, we found that in contrast to STZ-treated mice, the inhibition of muscle regeneration is much milder in Akita mice, which is likely explained by the residual insulin and long term compensatory adaptations that counter the mutation in the insulin 2 gene ${ }^{[24]}$, as compared with the rapid and complete ablation of pancreatic $\beta$-cells in STZ-treated mice.
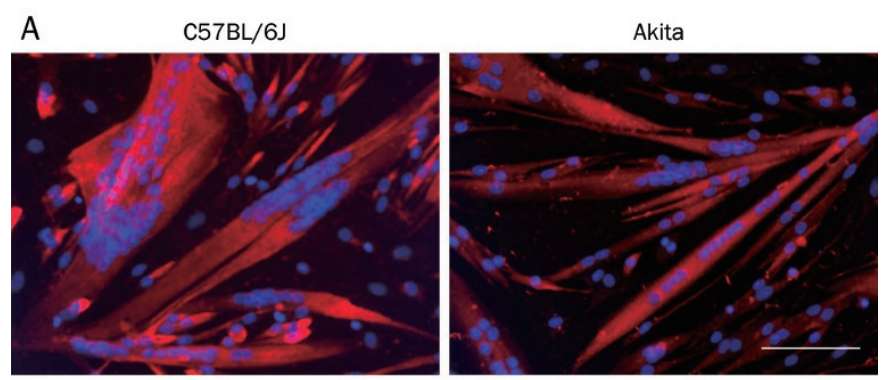

eMyHC/Hoechst
B

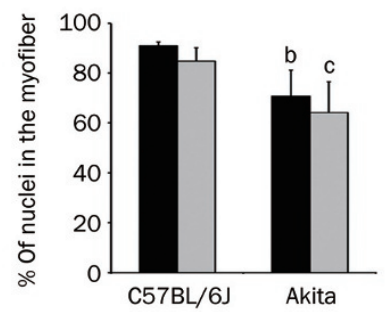

C

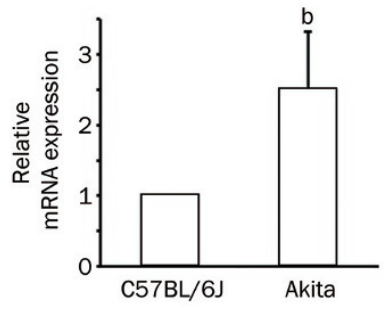

Figure 5. Satellite cell in vitro myogenic potential declines and myostatin mRNA levels increase in C57BL/6J-Ins2Akita mice. (A) Satellite cells were prepared from TA and Gastroc muscle of C57BL/7J and Akita mice at 3 $\mathrm{DPI}$, cultured in growth media for $48 \mathrm{~h}$ and then switched to differentiation media for $48 \mathrm{~h}$. Formation of de-novo eMyHC $\mathrm{Hy}^{+}$mytubes (red) was assayed by immunofluorescence. Hoechst (blue) labels all nuclei. Scale bar, $100 \mu \mathrm{m}$. (B) The ratio of nuclei in $\mathrm{eMyHC}^{+}$nuclei myotubes to total nuclei $\times 100$ (black bars), the ratio of the $\mathrm{eMyHC}^{+}$myotubes with than two nuclei to total nuclei $\times 100$ (gray bars) were quantified. ${ }^{\mathrm{b}} P<0.05$, and ${ }^{\mathrm{C}} P<0.01$ Akita as compared to $\mathrm{C} 57 \mathrm{BL} / 6 \mathrm{~J}$. Data are mean $\pm \mathrm{SD}$. (C) Myofibers were prepared from TA and Gastroc muscle of C57BL/6J and Akita mice at $3 \mathrm{DPI}$, lysed and used for qRT PCR with myostatin-specific primers. ${ }^{\mathrm{b}} P<0.05$ Akita as compared to $\mathrm{C} 57 \mathrm{BL} / 6 \mathrm{~J}$. Data are mean $\pm \mathrm{SD}$.

\section{Discussion}

This work is to demonstrate that deregulation of glucose metabolism immediately and severely suppresses the responses of muscle stem cells and thus directly incapacitates tissue regeneration, as established here in skeletal muscle, and likely applies to other tissues and their dedicated stem cells. In this regard it was shown that under ischemia, neural stem cells die from glucose deprivation rather than from oxygen deprivation $^{[26]}$. The conclusion that organ stem cells are likely to be acutely sensitive to low insulin/hyperglycemia/poor glucose metabolism implies that progressive tissue degeneration and poor wound healing, which are known manifestations of diabetic mellitus ${ }^{[3]}$, could be in large part caused by suppressed stem cell responses.

In agreement with the STZ results, the lack of muscle stem cell regenerative capacity was statistically significant at the onset of diabetes in Akita mice, even though the muscle regenerative capacity was diminished only mildly; with time a cumulative regenerative decline can lead to a significant loss of functional muscle tissue. At the level of molecular mechanism, this work establishes that in a hyperglycemic environment, muscle stem cells fail to engage in tissue repair largely because of increased signaling through the TGF $\beta$ receptor phosphorylation of Smad3, and induction of p15 gene expression, and that the regenerative capacity can be rescued in vivo by reducing TGF $\beta$ signaling through systemic administration of follistatin or an Alk5 inhibitor. The use of the Alk5 inhibitor confirmed that myostatin acts via the canonical TGF $\beta$ receptor pathway to inhibit muscle regeneration in STZ-treated hyperglycemic mice. Since follistatin is a more specific antagonist of myostatin, it could be potentially developed into a treatment for combating muscle wasting in patients with type 1 and type 2 diabetes mellitus, or to enhance tissue repair in such individuals after injury or surgery. Future work will determine whether it is the high blood glucose levels, the absence of insulin signaling, or both that result in the elevation of myostatin/TGF- $\beta$ receptor signaling, the up-regulation of p15 and the resulting decline in regenerative responses of muscle stem cells.

\section{Acknowledgements}

This work was supported by grants from the National Institutes of Health, USA R01 AG02725201 and Congressional Stimulus Fund Supplement, and California Institute for Regenerative Medicine RN1-00532-1.

\section{Author contribution}

Jaemin JEONG performed experiments, analyzed the data and co-wrote the manuscript; Michael J CONBOY designed experiments, analyzed the data and edited the manuscript; and Irina M CONBOY designed the study and wrote the manuscript.

\section{Supplementary information}

Supplementary information is available at the Acta Pharmacologica Sinica website. 


\section{References}

1 Furie K, Inzucchi SE. Diabetes mellitus, insulin resistance, hyperglycemia, and stroke. Curr Neurol Neurosci Rep 2008; 8: 12-9.

2 Venn BJ, Green TJ. Glycemic index and glycemic load: measurement issues and their effect on diet-disease relationships. Eur J Clin Nutr 2007; 61: S122-31.

3 Botusan IR, Sunkari VG, Savu O, Catrina Al, Grünler J, Lindberg S, et al. Stabilization of HIF-1alpha is critical to improve wound healing in diabetic mice. Proc Natl Acad Sci U S A 2008; 105: 19426-31.

4 Conboy IM, Rando TA. Aging, stem cells and tissue regeneration: lessons from muscle. Cell Cycle 2005; 4: 407-10.

5 Buckingham M, Montarras D. Skeletal muscle stem cells. Curr Opin Genet Dev 2008; 18: 330-6.

6 Smythe GM, Shavlakadze T, Roberts P, Davies MJ, McGeachie JK, Grounds MD. Age influences the early events of skeletal muscle regeneration: studies of whole muscle grafts transplanted between young (8 weeks) and old (13-21 months) mice. Exp Gerontol 2008; 43: 550-62.

7 Forster O, Rudas B. Ketosis in rats with streptozotocin-induced diabetes. Lancet 1969; 1: 1321-2.

8 Scheele C, Nielsen S, Kelly M, Broholm C, Nielsen AR, Taudorf S, et al. Satellite cells derived from obese humans with type 2 diabetes and differentiated into myocytes in vitro exhibit abnormal response to IL-6. PLoS One 2012; 7: e39657.

9 Chen Y, Cao L, Ye J, Zhu D. Upregulation of myostatin gene expression in streptozotocin-induced type 1 diabetes mice is attenuated by insulin. Biochem Biophys Res Commun 2009; 388: 112-6.

10 McCroskery S, Thomas M, Maxwell L, Sharma M, Kambadur R. Myostatin negatively regulates satellite cell activation and selfrenewal. J Cell Biol 2003; 162: 1135-47.

11 Vignaud A, Ramond F, Hourdé C, Keller A, Butler-Browne G, Ferry A. Diabetes provides an unfavorable environment for muscle mass and function after muscle injury in mice. Pathobiology 2007; 74: $291-$ 300.

12 Krause MP, Moradi J, Nissar AA, Riddell MC, Hawke TJ. Inhibition of plasminogen activator inhibitor-1 restores skeletal muscle regeneration in untreated type 1 diabetic mice. Diabetes 2011; 60: 1964-72.

13 Conboy IM, Conboy MJ, Smythe GM, Rando TA. Notch-mediated restoration of regenerative potential to aged muscle. Science 2003; 302: $1575-7$.

14 Conboy MJ, Conboy IM. Preparation of adult muscle fiber-associated stem/precursor cells. Methods Mol Biol 2010; 621: 149-63.

15 Conboy IM, Rando TA. Heterochronic parabiosis for the study of the effects of aging on stem cells and their niches. Cell Cycle 2012; 11: 2260-7.

16 Leiria LO, Mónica FZ, Carvalho FD, Claudino MA, Franco-Penteado CF, Schenka A, et al. Functional, morphological and molecular characterization of bladder dysfunction in streptozotocin-induced diabetic mice: evidence of a role for L-type voltage-operated $\mathrm{Ca}^{2+}$ channels. $\mathrm{Br}$ J Pharmacol 2011; 163: 1276-88.

17 Conboy IM, Conboy MJ, Wagers AJ, Girma ER, Weissman IL, Rando TA. Rejuvenation of aged progenitor cells by exposure to a young systemic environment. Nature 2005; 433: 760-4.

18 Brack AS, Conboy IM, Conboy MJ, Shen J, Rando TA. A temporal switch from notch to Wnt signaling in muscle stem cells is necessary for normal adult myogenesis. Cell Stem Cell 2008; 2: 50-9.

19 Conboy IM, Rando TA. Heterochronic parabiosis for the study of the effects of aging on stem cells and their niches. Cell Cycle 2012; 11: 2260-7.

20 Conboy IM, Yousef H, Conboy MJ. Embryonic anti-aging niche. Aging (Albany NY) 2011; 3: 555-63.

21 Goldstein JA, Kelly SM, LoPresti PP, Heydemann A, Earley JU, Ferguson $E L$, et al. SMAD signaling drives heart and muscle dysfunction in a Drosophila model of muscular dystrophy. Hum Mol Genet 2011; 20 : 894-904.

22 Trinh BQ, Barengo N, Naora H. Homeodomain protein DLX4 counteracts key transcriptional control mechanisms of the TGF- $\beta$ cytostatic program and blocks the antiproliferative effect of TGF- $\beta$. Oncogene 2011; 30: 2718-29.

23 Barber AJ, Antonetti DA, Kern TS, Reiter CE, Soans RS, Krady JK, et al. The Ins2Akita mouse as a model of early retinal complications in diabetes. Invest Ophthalmol Vis Sci 2005; 46: 2210-8.

24 Leroux L, Desbois P, Lamotte L, Duvillié B, Cordonnier N, Jackerott M, et al. Compensatory responses in mice carrying a null mutation for Ins1 or Ins2. Diabetes 2001; 50: S150-3.

25 Babad J, Geliebter A, DiLorenzo TP. T-cell autoantigens in the nonobese diabetic mouse model of autoimmune diabetes. Immunology 2010; 131: 459-65.

26 Horie N, Moriya T, Mitome M, Kitagawa N, Nagata I, Shinohara K. Lowered glucose suppressed the proliferation and increased the differentiation of murine neural stem cells in vitro. FEBS Lett 2004; 571: $237-42$.

27 Chen Y, Ye J, Cao L, Zhang Y, Xia W, Zhu D. Myostatin regulates glucose metabolism via the AMP-activated protein kinase pathway in skeletal muscle cells. Int J Biochem Cell Biol 2010; 42: 2072-81. 